\title{
Successful treatment of a patient with oligometastatic mesothelioma to the brain
}

Selena S. Li, BA, ${ }^{a}$ Desiree A. Steimer, MD, ${ }^{\mathrm{b}}$ Shannon Coy, MD, ${ }^{\mathrm{c}}$ and Raphael Bueno, MD, ${ }^{\mathrm{b}}$ Boston, Mass

From ${ }^{\mathrm{a} H a r v a r d}$ Medical School; and ${ }^{\mathrm{b}}$ Division of Thoracic Surgery and ${ }^{\mathrm{c}}$ Department of Pathology, Brigham and Women's Hospital, Boston, Mass.

Disclosures: Dr Bueno receives funding from Medgenome, Roche, Verastem, Merck, Gritstone, Epizyme, Siemens, and Celsius as well as grants from the National Cancer Institute and Department of Defense for other research projects. All other authors reported no conflicts of interest.

The Journal policy requires editors and reviewers to disclose conflicts of interest and to decline handling or reviewing manuscripts for which they may have a conflict of interest. The editors and reviewers of this article have no conflicts of interest.

Received for publication May 19, 2020; revisions received May 19, 2020; accepted for publication May 20, 2020; available ahead of print May 30, 2020.

Address for reprints: Desiree A. Steimer, MD, Division of Thoracic Surgery, Brigham and Women's Hospital, 75 Francis St, Boston, MA 02115 (E-mail: dsteimer@bwh.harvard.edu).

JTCVS Techniques 2020;3:358-60

2666-2507

Copyright $\odot 2020$ The Authors. Published by Elsevier Inc. on behalf of The American Association for Thoracic Surgery. This is an open access article under the CC BY-NC-ND license (http://creativecommons.org/licenses/bync-nd/4.0/).

https://doi.org/10.1016/j.xjtc.2020.05.019

Mesothelioma is a rare but aggressive malignancy, previously thought to only spread through local invasion. ${ }^{1,2}$ However, autopsy studies identified distant metastases in $54 \%$ of cases, with intracranial lesions representing only $3 \%$ of patients. ${ }^{1}$ Due to the low incidence of intracranial metastasis, there is no consensus on management for these patients. ${ }^{1-3}$ We report a case of pleural mesothelioma treated with pleurectomy and adjuvant chemotherapy that developed isolated recurrence to the brain 2 years after resection.

\section{CLINICAL SCENARIO}

A 48-year-old man with history of asbestos exposure was hospitalized for appendicitis, and an incidental right pleural effusion was noted during workup. Dedicated chest imaging demonstrated right pleural thickening; pleural biopsy was performed and diagnosed epithelioid type mesothelioma. Staging workup included positron emission tomographycomputed tomography (PET-CT) and magnetic resonance imaging (MRI) of the chest, which revealed localized disease within the right hemithorax and no distant metastasis. There was no evidence of nodal involvement by mediastinoscopy.

The patient underwent radical pleurectomy, decortication, and intraoperative heated chemotherapy with cisplatin and gemcitabine. Final pathologic staging was pT3NO, and he completed 4 cycles of adjuvant chemotherapy (cisplatin and pemetrexed). Routine surveillance was performed every 4 months with CT chest; he had no evidence of disease recurrence.

Two years after pleurectomy, he presented to the emergency department with word-finding difficulty and slurred speech. Examination noted new right facial droop and mild dysarthria. CT imaging showed a single lesion in the

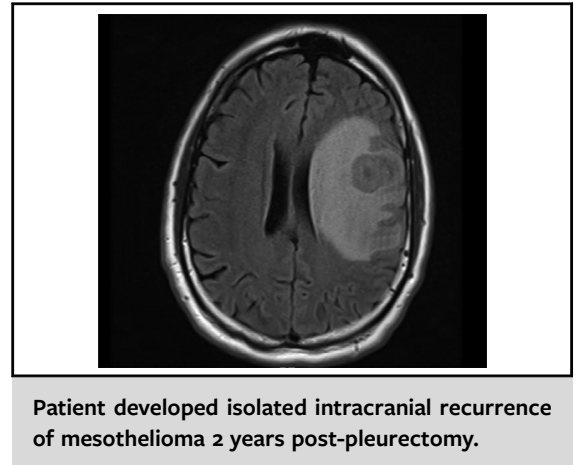

CENTRAL MESSAGE

As long-term survival from mesothelioma improves with aggressive multimodality treatment, we anticipate a shift in the recurrence pattern from local to distant sites of failure.

See Commentaries on pages 361,363 , and 365

left frontal lobe measuring $2.4 \times 2.2 \mathrm{~cm}$. MRI of the brain demonstrated vasogenic edema and $6 \mathrm{~mm}$ of midline shift (Figure 1). Restaging imaging was performed and negative for other sites of disease.

$\mathrm{He}$ underwent a left craniotomy and resection of the mass. Pathology confirmed epithelioid mesothelioma; there was no evidence of de-differentiation to biphasic or sarcomatoid subtype (Figure 2). He had an uneventful postoperative course with no residual neurologic symptoms. Due to close margins, he completed stereotactic radiation (25 Gy) to the surgical bed one month after resection.

Current surveillance plan includes MRI of the brain and $\mathrm{CT}$ of the chest every 6 months. He is 5.5 years postpleurectomy and 3.5 years since treatment of oligometastatic disease; he has no evidence of disease recurrence at time of reporting. Consent was obtained from the patient to publish this work.

\section{DISCUSSION}

Our case highlights the successful treatment of an intracranial recurrence from pleural mesothelioma. Although mesothelioma recurrence is common, the typical pattern 


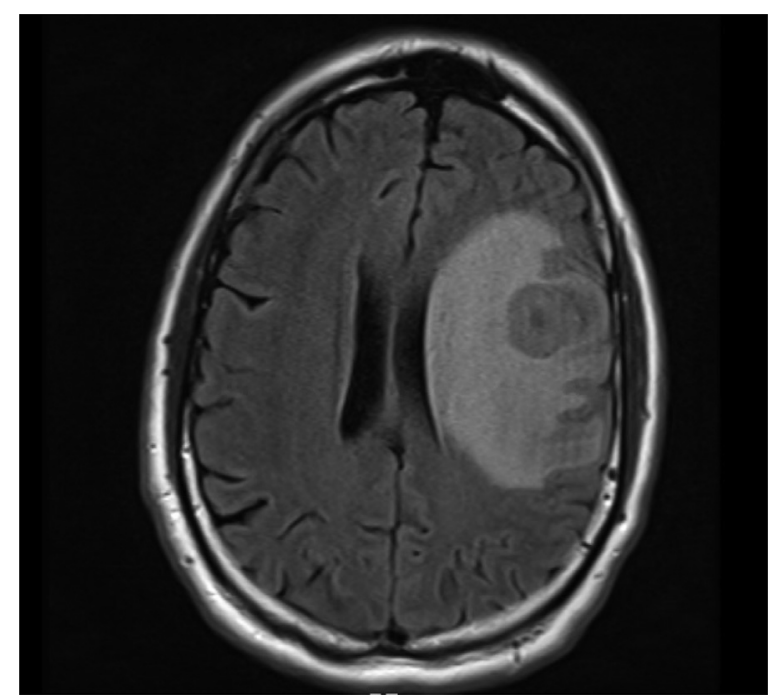

FIGURE 1. Magnetic resonance imaging of the brain (T2-weighted) in a patient with history of epithelioid type mesothelioma presenting with dysarthria and right facial droop. Axial image demonstrates solitary left frontal lobe lesion with associated vasogenic edema and midline parenchymal shift concerning for mesothelioma recurrence. favors ipsilateral hemithorax and mediastinum (54\%) over distant sites $(5 \%){ }^{4}$ Isolated recurrence of the chest wall treated with surgical resection has been shown to extend the overall survival in these patients. ${ }^{5}$ The survival benefit was more apparent in patients with long disease-free intervals before development of recurrence; this was true for epithelioid and biphasic subtypes. ${ }^{5}$ We suspect contributing factors to the survival of our patient were the 2-year duration before recurrence development and control of the primary and secondary sites of disease.

Intracranial metastasis from mesothelioma is rare and usually diagnosed at autopsy. ${ }^{1-3}$ Most patients do not exhibit neurologic symptoms, leading to the low detection rate. $^{1,2}$ Autopsy studies suggest that few patients have isolated brain lesions and more frequently have diffuse metastatic disease, contributing to their poor survival. ${ }^{1,3}$ Analysis of 59 patients with mesothelioma brain metastases compiled from case reports revealed $53 \%$ of these patients had sarcomatoid subtype as their primary tumor. ${ }^{2}$

Brain imaging is not routinely obtained as part of the initial staging workup or postresection surveillance for patients with mesothelioma at our institution. Due to the low
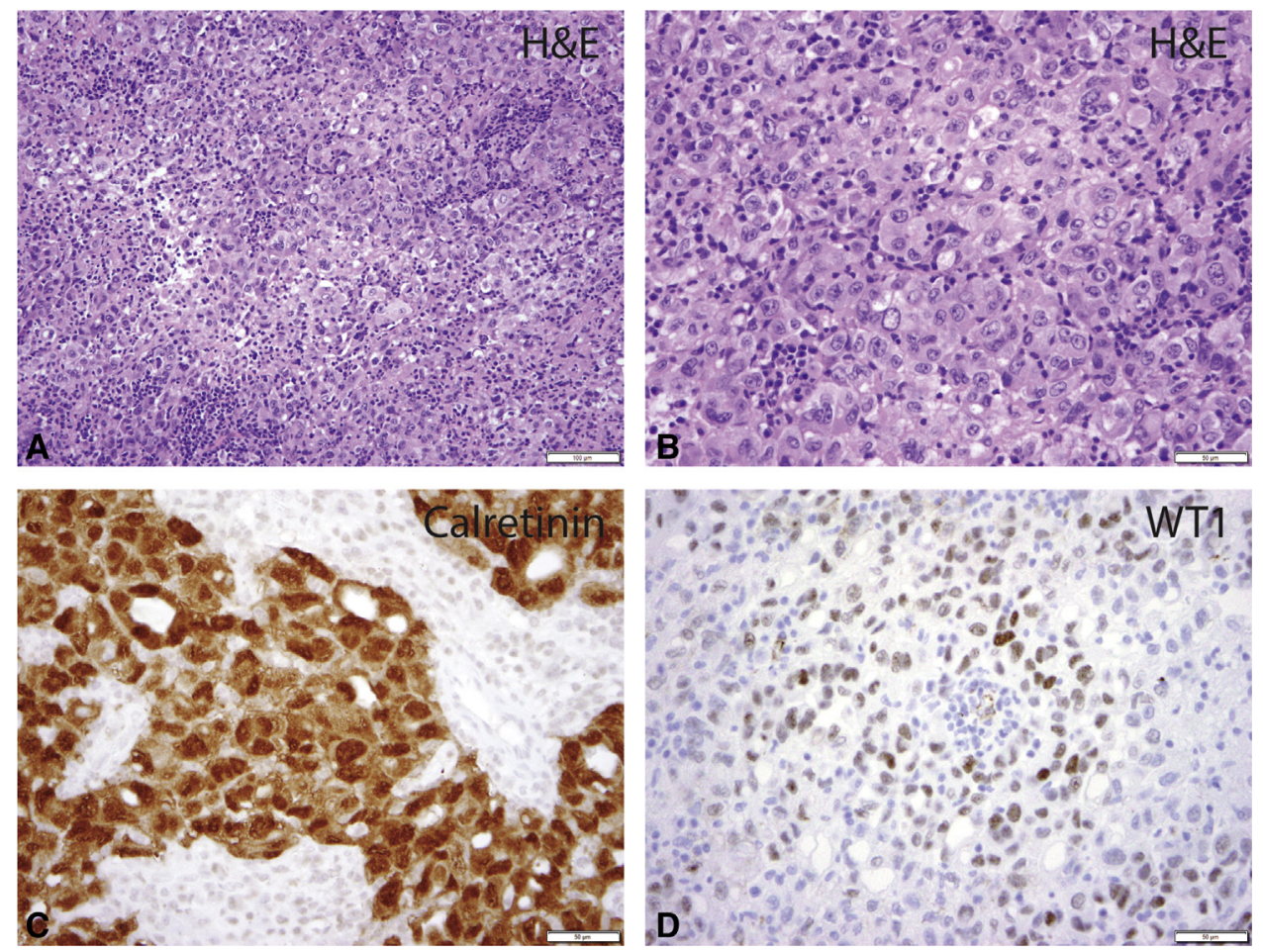

FIGURE 2. Histopathologic examination of H\&E-stained sections from left frontal lobe mass showing sheets and nests of atypical epithelioid cells with moderate nuclear pleomorphism, predominantly vesicular chromatin, variably prominent nucleoli, and abundant eosinophilic to clear cytoplasm (A, B). Immunophenotypically, the atypical cells showed (C) strong and diffuse nuclear and cytoplasmic expression of calretinin and (D) weak-to-moderate nuclear expression of WT1, consistent with the diagnosis of metastatic mesothelioma, epithelioid subtype. Pathologic analysis confirms histology similar to the primary tumor; there was no evidence of de-differentiation to biphasic or sarcomatoid subtype. Scale bars: $100 \mu \mathrm{m}$ (A), $50 \mu \mathrm{m}$ (B-D). $H \& E$, Hematoxylin and eosin. 
prevalence of intracranial recurrences, we do not advise routine brain imaging for postoperative surveillance. This patient is 1 of more than 150 long-term ( $>5$ years) survivors at our program and is the first to develop an intracranial metastasis. We recommend having a low threshold for CT or MRI brain in any mesothelioma patient with new neurologic complaints. Given the 2-fold increase of brain metastases in patients with sarcomatoid mesothelioma, ${ }^{2}$ brain imaging at time of initial staging or as part of their surveillance may be warranted.

There is no consensus on treatment of oligometastatic disease for mesothelioma, especially for intracranial lesions, given their low incidence. ${ }^{1-3}$ We have observed improved survival with salvage chest wall resections for recurrent disease $^{5}$ and strongly believe that resectable recurrences should be treated aggressively. With future advancements in treatment and better local control of disease, we anticipate a shift in the recurrence pattern and predict more distant failures occurring as presented in this case.
We propose that isolated recurrences (brain, adrenal, liver) be discussed in multidisciplinary conference and patients at least 1 year post-pleurectomy and without other sites of disease should be offered resection and/or radiation.

\section{References}

1. Falconieri G, Grandi G, DiBonito L, Bonifacio-Gori D, Giarelli L. Intracranial metastases from malignant pleural mesothelioma. Report of three autopsy cases and review of the literature. Arch Pathol Lab Med. 1991;115:591-5.

2. Miller AC, Miettinen M, Schrump DS, Hassan R. Malignant mesothelioma and central nervous system metastases. Report of two cases, pooled analysis, and systematic review. Ann Am Thorac Soc. 2014;11:1075-81.

3. Kitai R, Kabuto M, Kawano H, Uno H, Kobayashi H, Kubota T. Brain metastasis from malignant mesothelioma—case report. Neurol Med Chir (Tokyo). 1995;35: $172-4$.

4. Baldini EH, Richards WG, Gill RR, Goodman BM, Winfrey OK, Eisen HM, et al. Updated patterns of failure after multimodality therapy for malignant pleural mesothelioma. J Thorac Cardiovasc Surg. 2015;149:1374-81.

5. Burt BM, Ali SO, DaSilva MC, Yeap BY, Richards WG, Baldini EH, et al. Clinical indications and results after chest wall resection for recurrent mesothelioma. $J$ Thorac Cardiovasc Surg. 2013;146:1373-80. 